\title{
DESENVOLVIMENTO DE UM FOTÔMETRO DE BAIXO CUSTO PARA ANALISAR NÍVEIS DE NITRATOS EM ÁGUAS SUBTERRÂNEAS
}

\author{
LEITE MONIQUE J. L. ${ }^{1}$, FILHO ADEMAR ${ }^{1}$, BARROS IALY S. ${ }^{2}$, SILVA JOSIVAN P. ${ }^{2}$ e SOUZA \\ THIBÉRIO P. C. ${ }^{1,2}$ \\ ${ }^{1}$ Universidade Federal Rural de Pernambuco, Departamento de Engenharia de Alimentos \\ ${ }^{2}$ Universidade Federal de Pernambuco, Departamento de Engenharia Química \\ E-mail para contato: thiberio_souza@hotmail.com
}

\begin{abstract}
RESUMO - O uso de água proveniente de poços artesanais é uma alternativa para os locais onde não existe água encanada. $\mathrm{O}$ nitrogênio pode ser encontrado nas águas nas formas de nitrogênio orgânico, amoniacal, nitrito e nitrato. Os nitratos são tóxicos, causando uma doença denominada metahemoglobinemia infantil, que é letal para crianças. O uso de diodos emissores de luz (LED) juntamente com os Light Dependent Resistor (LDR), tem sido amplamente utilizados na construção de fotômetros portáteis. O fotômetro é uma ferramenta útil em análises, pois o mesmo oferece resultados quantitativos do analito a ser investigado. Neste trabalho, desenvolveu-se um fotômetro de baixo custo, para analisar níveis de nitratos em águas subterrâneas. Utilizou-se um microcontrolador do tipo ATMEGA8-16PC para o processamento de dados junto a um software desenvolvido para o equipamento. Os resultados obtidos foram comparados com um espectrofotômetro comercial de bancada, comprovando assim a eficácia do trabalho.
\end{abstract}

\section{INTRODUÇÃO}

As águas subterrâneas são utilizadas para o abastecimento doméstico nas áreas da zona urbana e rural, onde não existe água encanada proveniente de estações de tratamento. A passagem de nitrogênio de formas orgânicas para formas inorgânicas denomina-se mineralização, que ocorre pelas seguintes etapas: $\mathrm{N}$-orgânico; $\mathrm{N}$-amínico; $\mathrm{N}$-amoniacal; $\mathrm{N}$-nitrito; $\mathrm{N}$-nitrato. Diversas são as fontes de nitrogênio na água natural, os esgotos sanitários constituem a principal fonte de nitrogênio devido ao depósito de nitrogênio orgânico proveniente de proteínas e nitrogênio amoniacal decorrente da hidrolise da uréia na água. Arruda e colaboradores (2007) afirmam que, em pesquisas realizadas anteriormente no ano de 2004 (ARRUDA et al., 2007) na cidade de Garanhuns-PE, detectou-se a contaminação por Nitratos e Nitritos além do permitido pela legislação vigente. O Art. 37 da Portaria $n^{\circ}$ 2.914, de 12 de dezembro de 2011, estabelece como valor máximo permitido uma concentração de $10 \mathrm{mg} / \mathrm{L}$ de nitratos e $1 \mathrm{mg} / \mathrm{L}$ para nitritos.

Nitratos e nitritos podem causar problemas de ordem fisiológica, principalmente em crianças até 08 anos, que é a perda da capacidade de oxigenação do sangue (metemoglobinemia) (ARRUDA et al., 2007). No entanto Alaburda \& Nishihara (1998), afirmam que o nitrito, quando 
presente na água de consumo humano, tem um efeito mais rápido e pronunciado do que o nitrato. Se o nitrito for ingerido diretamente, pode ocasionar metemoglobinemia independente da faixa etária do consumidor. Os métodos espectroscópicos de análise química são baseados na medição da quantidade de radiação eletromagnética produzida ou absorvidos por um analito de interesse. As interações da radiação eletromagnética com a matéria são objeto de estudo em diversas áreas, como na área farmacêutica, na quantificação de princípios ativos, na área ambiental, na determinação de macronutrientes e elementos-traço em matrizes ambientais e na área industrial, na determinação de compostos de interesse nutricional.

Devido a isso, é interessante o uso de técnicas capazes de analisar o nível de nitratos e nitritos em águas nas comunidades carentes de serviços especializados. Uma das técnicas usadas para a determinação dos níveis de nitratos e nitritos em águas é a espectroscopia na região do visível (MARCO et al., 2006). Por outro lado esta técnica necessita de mão de obra especializada além do uso de instrumentos apropriados. Os equipamentos mais usados são o fotômetro e o espectrofotômetro. Estes equipamentos, apesar de usarem uma técnica simples, são de custo expressivo e inviáveis para que uma comunidade carente possa adquiri-los para seu uso no dia a dia. Contudo, existem cada vez mais, o uso de tecnologias alternativas capazes de desempenhar esta função a custo muito reduzido. Entre elas está o de poder confeccionar um próprio fotômetro com materiais simples como LED's (light-emitting diode) e LDR's (Light Dependent Resistor) (FÁBIO, 2010). O objetivo principal deste trabalho foi desenvolver um fotômetro UV-Visível de baixo custo com tecnologia embarcada e software próprio para medir níveis de nitratos em águas subterrâneas.

\section{MATERIAIS E MÉTODOS}

Para a montagem do equipamento, foi utilizado como componentes principais um Light Emitting Diode como fonte de emissão de luz na faixa do visível uma vez que é possível modular os comprimentos de onda emitidos, um e Light Dependent Resistor como detector de luminosidade e um microcontrolador do tipo ATMEGA8-16PC para o processamento das informações e varredura do processo conforme mostrado na Figura 1.

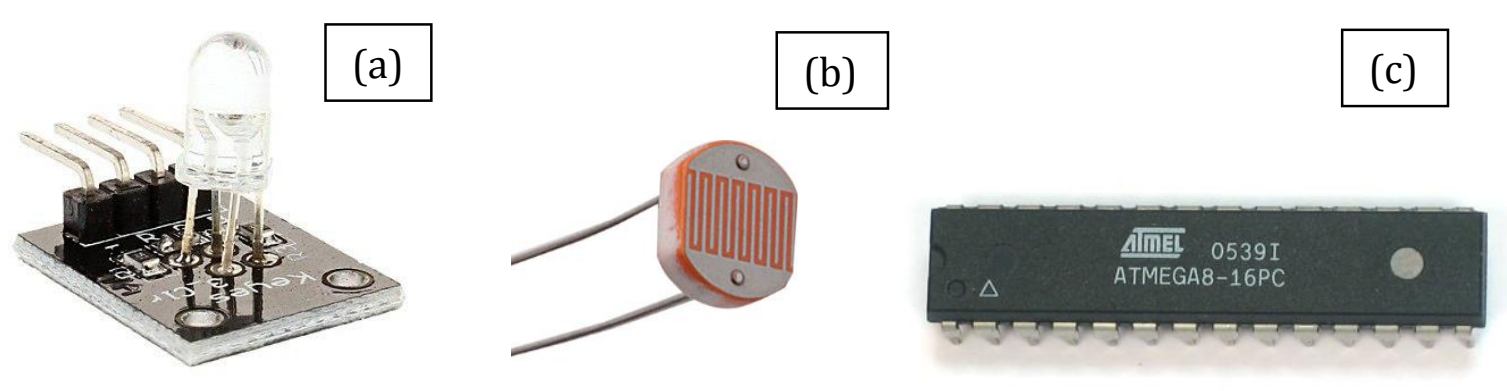

Figura 1 - Componentes Light Emitting Diode LED/RGB (a), Light Dependent Resistor LDR (b) e Microcontrolador ATMEGA8-16PC (c). 
Para a construção do corpo do equipamento, foi utilizado uma caixa plástica com tampa de encaixe na qual foram pintados com tinta preta fosco a fim de se evitar a interferência luminosa provocada tanto pelo meio externo quanto por reflexão interna do próprio equipamento (Figura 2-a). Foi desenvolvido uma porta de acesso para a introdução da cubeta com as amostras (Figura 2-b) de forma que possa ser usado o equipamento continuamente, bastando apenas retirar ou inserir a cubeta.

Todos os componentes foram montados na estrutura conforme visto na Figura 2-c e Figura 2-d.

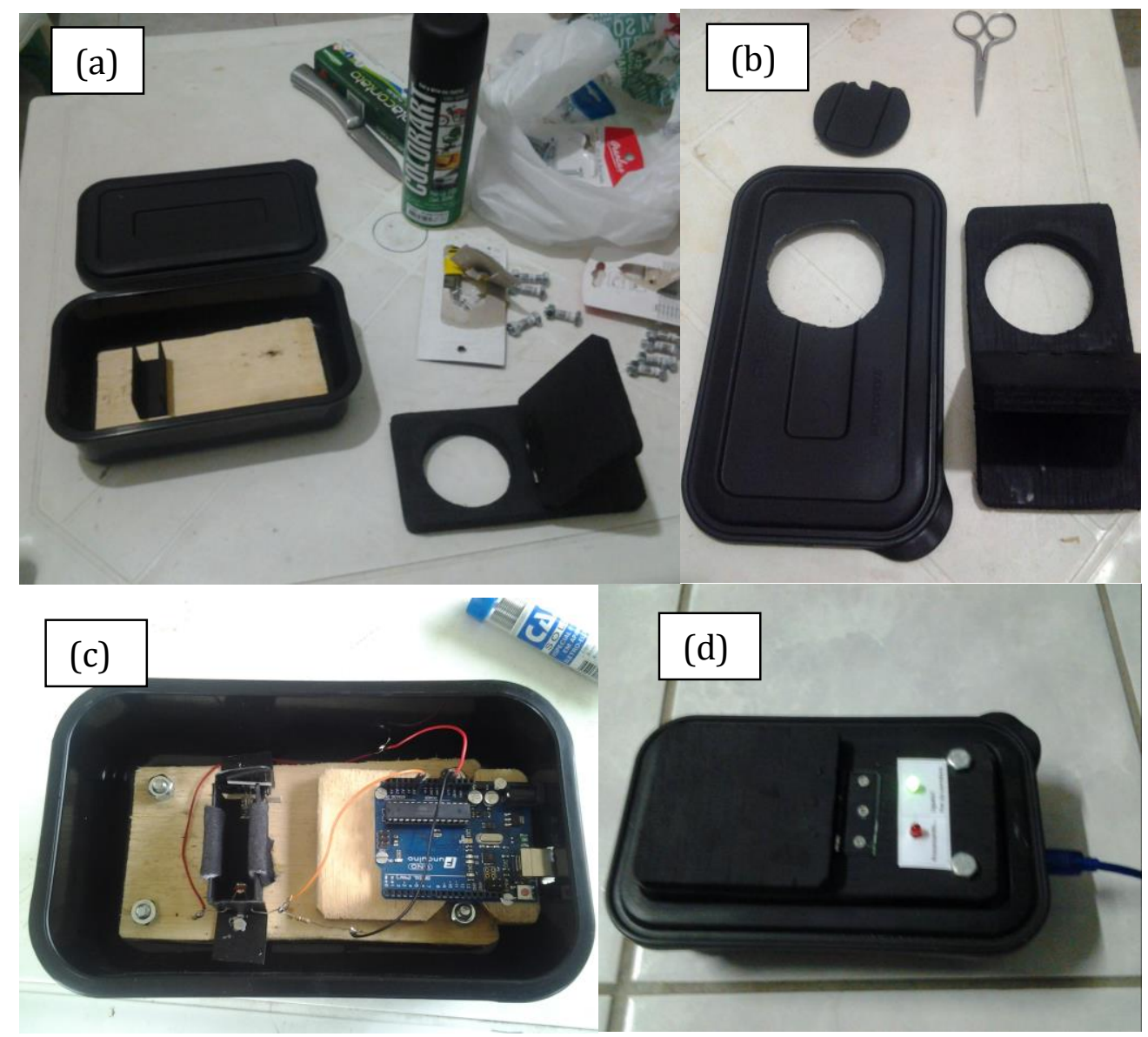

Figura 2 - Montagem do Fotômetro com pintura preto fosco (a), Porta de acesso à cubeta (b), montagem dos componentes internos (c) e vista do equipamento finalizado (d).

Em seguida, foi desenvolvido um algoritmo (linguagem C) capaz de realizar a varredura dos comprimentos de onda na região do visível. Procurou-se varrer a faixa que vai do Violeta à região do Vermelho. O algoritmo modula a intensidade do Light Emitting Diode - Red Green Blue fazendo com que o mesmo emita energia no comprimento de onda pré-estabelecido para a análise dos resultados.

Também é possível medir os dados lidos pelo Light Dependent Resistor onde os quais são enviados através de comunicação USB para um computador pessoal. O programa foi compilado e salvo no microcontrolador do equipamento deixando automático todo o processo de análise das amostras 
sendo apenas necessário ligar o fotômetro a um computador pessoal.

O valor pago para desenvolver o equipamento foi de $\mathrm{R} \$ 85,60$ incluindo todos os componentes, corpo físico e materiais de apoio à confecção do fotômetro. Os gastos do projeto estão muito além do custo médio de um equipamento comercial orçado em $\mathrm{R} \$ 3.354,36$.

Em seguida, foi necessário realizar a calibração do equipamento. Para isso, o fotômetro em operação foi ligado a um notebook como pode ser visto na Figura 3-a. A Figura 3-b mostra a varredura dos espectros realizada pelo algoritmo na qual será tratada com uma programação específica para os comprimentos de onda lidos pelo instrumento.

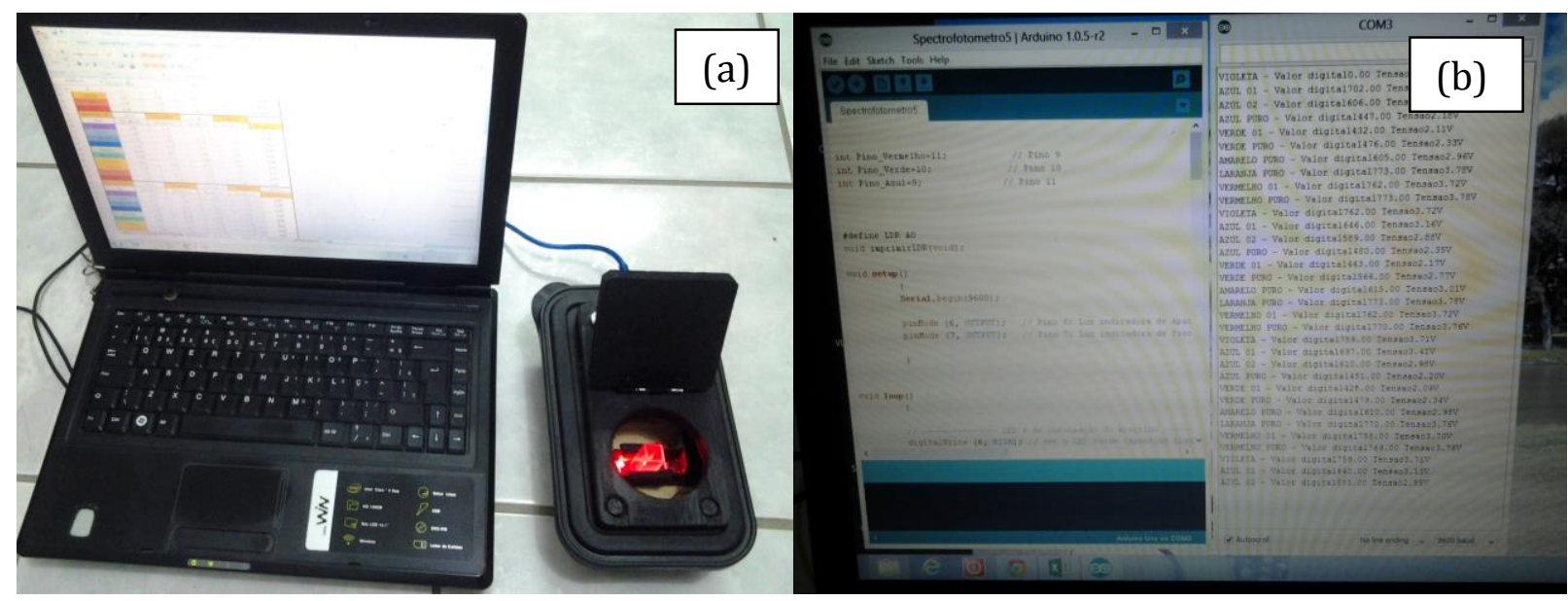

Figura 3 - Fotômetro ligado a um notebook (a). Varredura dos sinais (b).

O Art. 37 da Portaria n ${ }^{\circ}$ 2.914, de 12 de dezembro de 2011, estabelece como valor máximo permitido uma concentração de $10 \mathrm{mg} / \mathrm{L}$ de nitratos. Em virtude disto, foi preparado 3 soluções conhecidas de Nitrato de Sódio sendo uma um nível abaixo (-1) e a outra um nível acima (+1) a fim de se obter uma curva padrão. As soluções preparadas foram de $5 \mathrm{mg} / \mathrm{L}, 10 \mathrm{mg} / \mathrm{L} \mathrm{e} 15 \mathrm{mg} / \mathrm{L}$ conforme mostrado na Figura 4. O método para análise de nitrato por espectrofotometria para águas potáveis seguiu o Manual de Procedimentos de Amostragem e Análise Físico-Química de Água (LUCILIA et al., 2011) e o método tentativo na $13^{a}$ edição do Standard Methods for Examination of Water and Wastewater de 1971.

O Script para a análise do resultado do fotômetro foi desenvolvido em MATLAB®. O resultado da concentração é obtido através de ajustes polinomiais de sexta ordem onde cada faixa de cor gera uma curva de intensidade do sinal versus concentração. Para se gerar dados confiáveis, foram utilizadas as amostras padrão com teores de nitrato conhecidos (5 mg/L, $10 \mathrm{mg} / \mathrm{L} \mathrm{e} 15 \mathrm{mg} / \mathrm{L})$. Para a calibração do equipamento, o script gera um polinômio para cada comprimento de onda gerado pelas amostras conhecidas, que é tratado como o padrão, como visto na Figura 5. Para cada nível de energia absorvido, o programa verifica qual o comprimento de onda mais significativo fazendo assim o melhor ajuste. Com isso, o programa consegue ser calibrado em função de amostras conhecida e obtidas em laboratório. Por fim, foi realizada coleta de amostras em 
Garanhuns/PE e localidades próximas a cidade de Capoeiras/PE com o objetivo de se verificar o nível de nitrato contido nas mesmas.

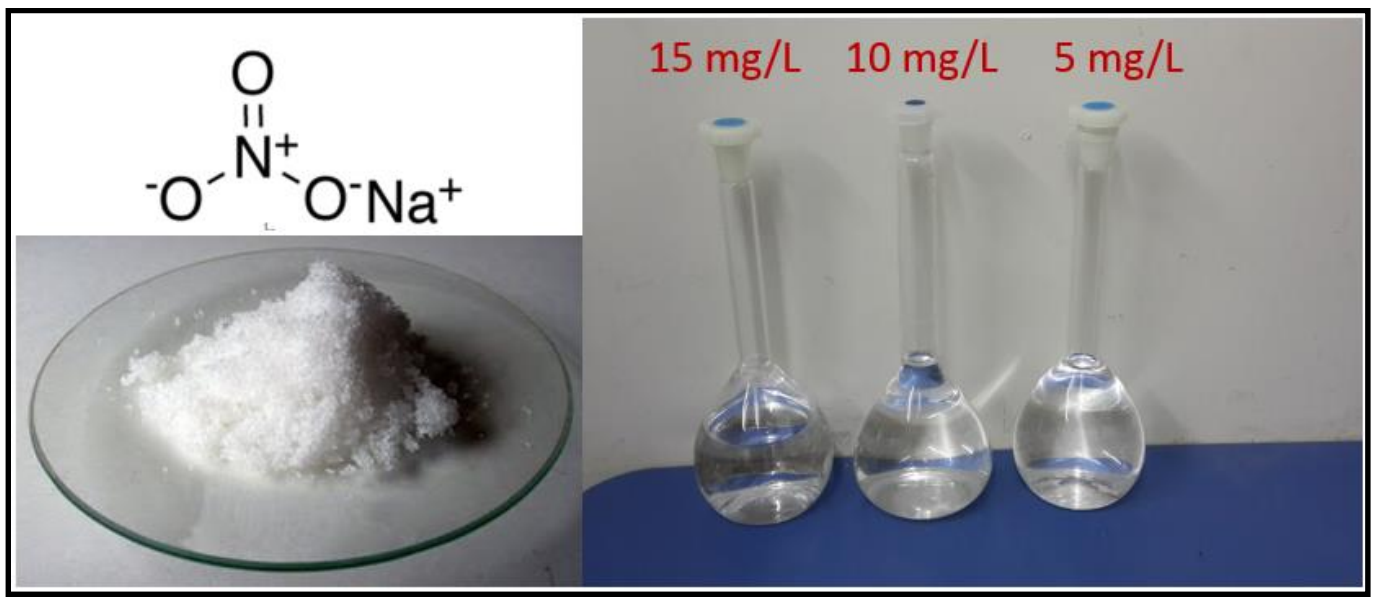

Figura 4 - Solução de Nitrato de Sódio a 5 mg/L, 10 mg/L e 15 mg/L.

Para analisar uma amostra desconhecida o script procura qual a concentração que tem o mesmo sinal de saída da amostra, resolvendo uma equação polinomial de $3^{\circ}$ grau, onde apenas uma das 3 soluções é dada como correta. A solução correta é a que não apresenta parte imaginária e que se encontra na faixa de concentração dos padrões conhecidos obtidos em laboratório. Com isso o Script demonstra a concentração estimada da amostra desconhecida plotando um gráfico de superfície. A Figura 5 mostra o ajuste padrão para as concentrações de nitrato em função do sinal.

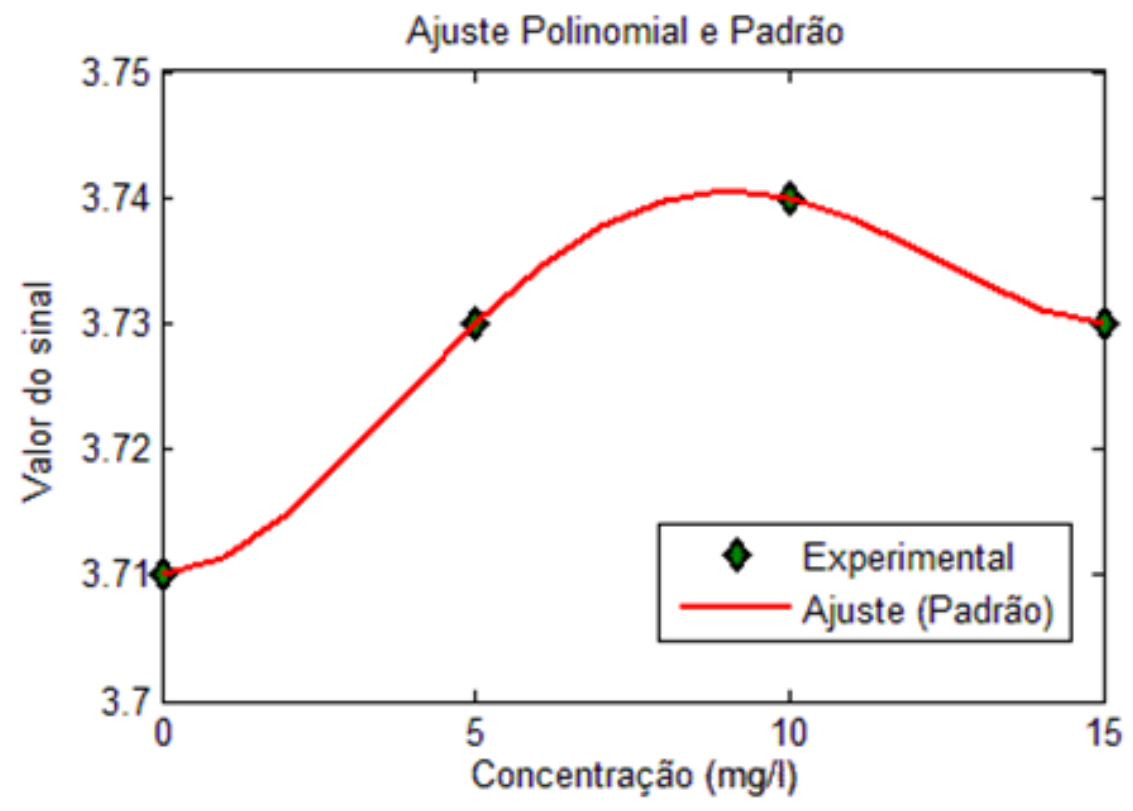

Figura 5 - Curva do ajuste padrão para as concentrações de nitrato em função do sinal. 


\section{RESULTADOS E DISCUSSÃO}

Os resultados das varreduras feitas nas soluções de Nitrato com 5 mg/L, 10 mg/L e 15 mg/L podem ser vistos na Tabela 1.

Tabela 1: Resultado das tensões em volts obtida pelas varreduras nas amostras de água com concentração de Nitrato de $5 \mathrm{mg} / \mathrm{L}, 10 \mathrm{mg} / \mathrm{L}$ e $15 \mathrm{mg} / \mathrm{L}$.

\begin{tabular}{c|ccc}
\hline Comprimento de Onda & Tensão $(5 \mathrm{mg} / \mathrm{L})$ & Tensão $(10 \mathrm{mg} / \mathrm{L})$ & Tensão $(15 \mathrm{mg} / \mathrm{L})$ \\
\hline $400 \mathrm{~nm}$ & $\mathbf{3 , 6 8}$ & $\mathbf{3 , 6 8}$ & $\mathbf{3 , 6 8}$ \\
$410 \mathrm{~nm}$ & $\mathbf{3 , 2 6}$ & $\mathbf{3 , 2 7}$ & $\mathbf{3 , 2 6}$ \\
$430 \mathrm{~nm}$ & $\mathbf{2 , 9 2}$ & $\mathbf{2 , 9 3}$ & $\mathbf{2 , 9 1}$ \\
$440 \mathrm{~nm}$ & $\mathbf{2 , 2 8}$ & $\mathbf{2 , 2 9}$ & $\mathbf{2 , 2 8}$ \\
$500 \mathrm{~nm}$ & $\mathbf{2 , 1 6}$ & $\mathbf{2 , 1 7}$ & $\mathbf{2 , 1 5}$ \\
$520 \mathrm{~nm}$ & $\mathbf{2 , 5 3}$ & $\mathbf{2 , 5 4}$ & $\mathbf{2 , 5 3}$ \\
$580 \mathrm{~nm}$ & $\mathbf{2 , 9 4}$ & $\mathbf{2 , 9 5}$ & $\mathbf{2 , 9 4}$ \\
$590 \mathrm{~nm}$ & $\mathbf{3 , 7 3}$ & $\mathbf{3 , 7 4}$ & $\mathbf{3 , 7 3}$ \\
$650 \mathrm{~nm}$ & $\mathbf{3 , 6 8}$ & $\mathbf{3 , 6 8}$ & $\mathbf{3 , 6 8}$ \\
$700 \mathrm{~nm}$ & $\mathbf{3 , 7 3}$ & $\mathbf{3 , 7 3}$ & $\mathbf{3 , 7 3}$ \\
\hline
\end{tabular}

Em seguida foi possível plotar um gráfico de Absorbância em função do comprimento de onda para as concentrações de $5 \mathrm{mg} / \mathrm{L}, 10 \mathrm{mg} / \mathrm{L}$ e $15 \mathrm{mg} / \mathrm{L}$ das soluções conhecidas (Figura 6).

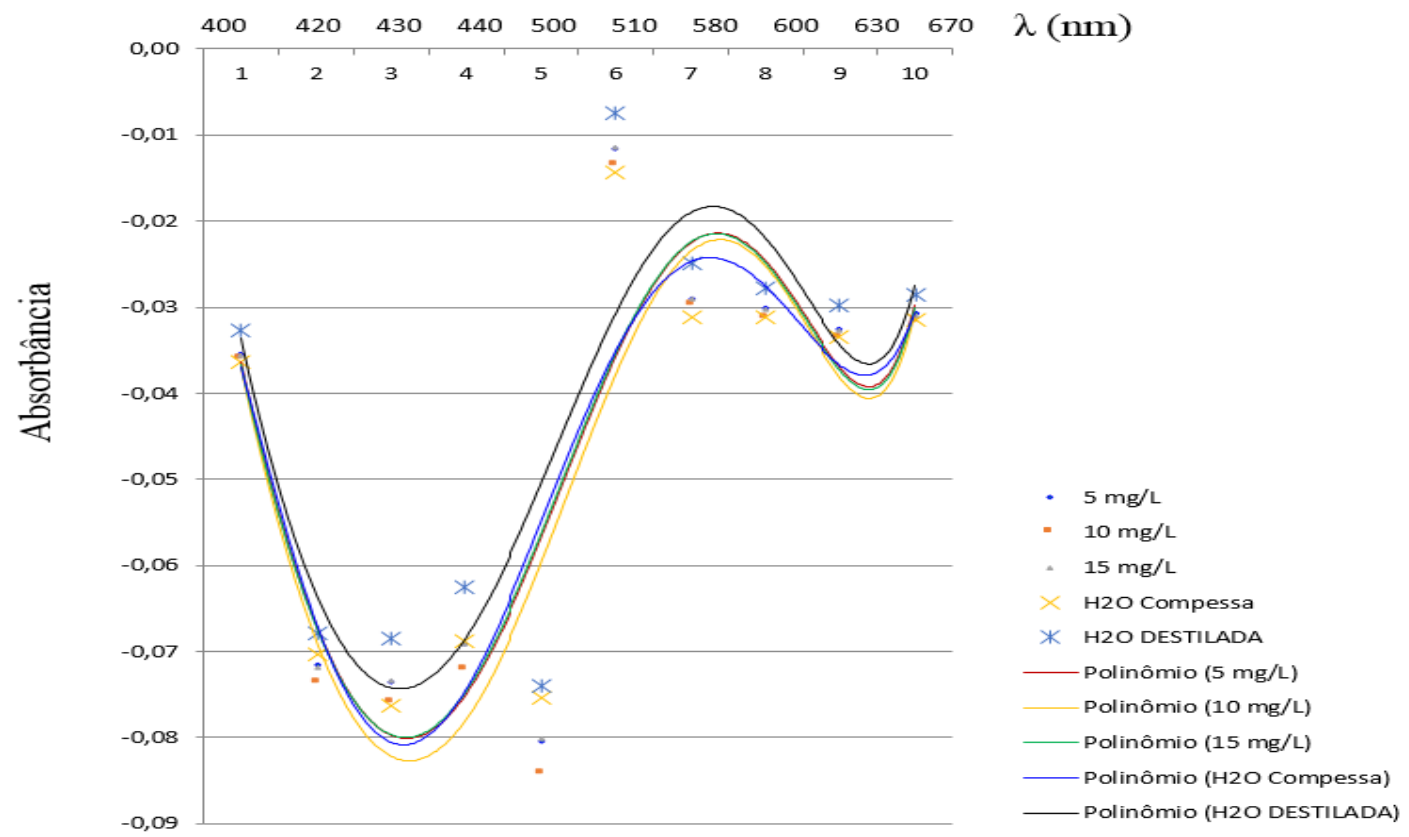

Figura 6 - Gráfico da Absorbância pelo comprimento de onda das soluções de Nitrato. 
A Figura 7 mostra a resposta obtida pelo instrumento em função da varredura do espectro obtendo, com isso, a concentração de Nitrato na amostra.

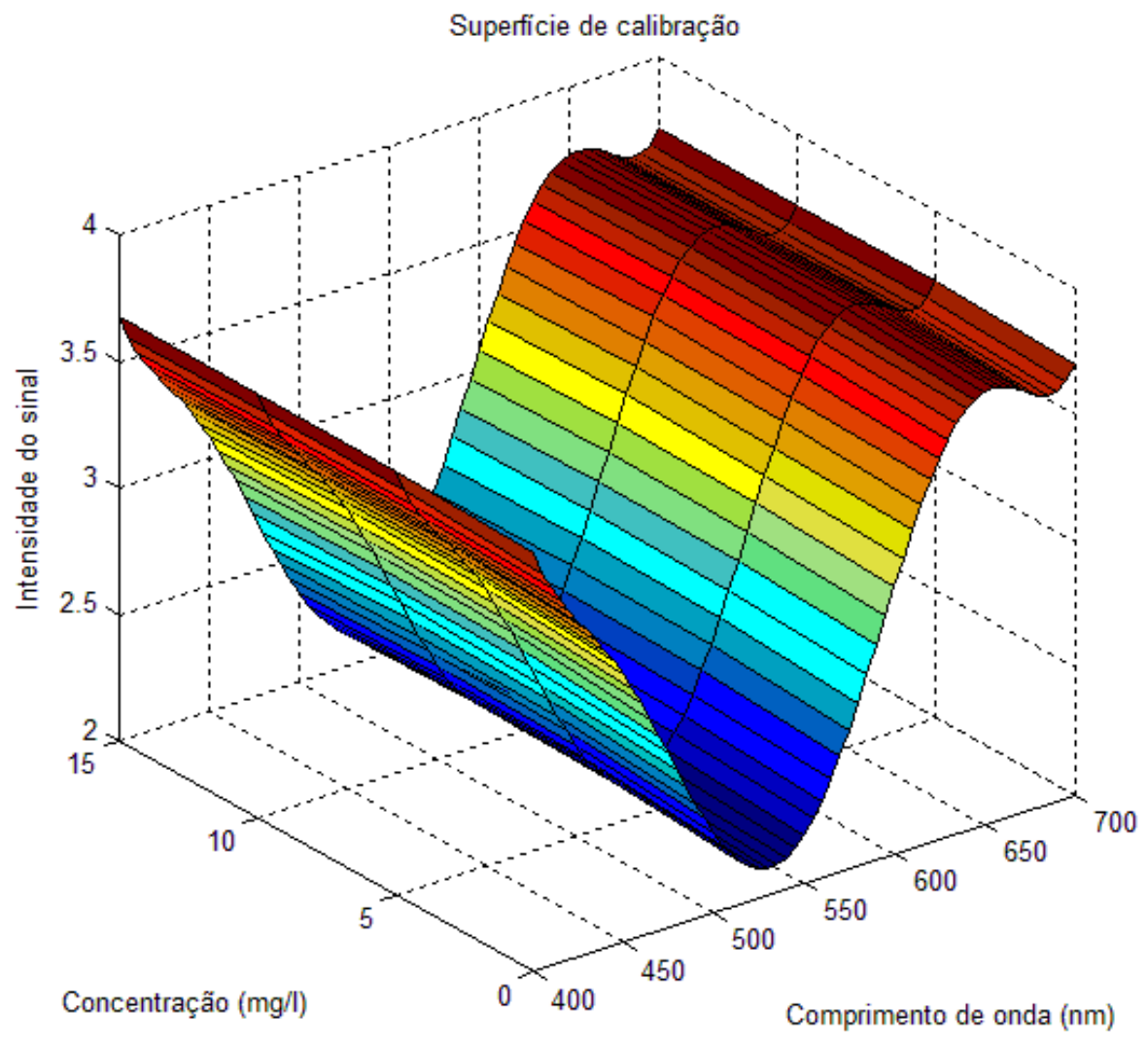

Figura 7 - Gráfico de superfície de intensidade do sinal lido pelo instrumento em função da concentração de Nitrato e do comprimento de onda associado.

Foram realizados testes nas águas utilizadas para consumo proveniente de fontes naturais, coletadas nas comunidades oriundas da região satélite da cidade de Garanhuns/PE e Capoeiras/PE. Os resultados da concentração, em mg/L, de Nitrato contido nas amostras de água podem ser vistos na Tabela 2 mostrada a seguir.

Tabela 2: Resultado das concentrações de Nitrato presente nas águas em mg/L.

\begin{tabular}{c|ccccc}
\hline \multicolumn{1}{c}{ Análise } & Água Compesa $^{*}$ & Água Potável & Região1 & Região 2 & Região 3 \\
\hline Laboratório & 4,27 & 1,42 & 2,11 & 0,62 & 0,49 \\
Fotômetro & 4,11 & 1,39 & 2,51 & 0,50 & 0,45 \\
\hline
\end{tabular}

* Companhia Pernambucana de Saneamento. 


\section{CONCLUSÃO}

O resultado obtido pelo fotômetro desenvolvido na Universidade Federal Rural de Pernambuco foi bastante satisfatório, uma vez que os níveis de Nitrato lidos pelo instrumento condizem com os resultados obtidos em laboratório. Com um custo de montagem orçado em cerca de $2,5 \%$ do valor de um instrumento comercial, o fotômetro sugerido neste trabalho atende as necessidades de um equipamento de baixo custo sendo acessível a qualquer pessoa.

\section{REFERÊNCIAS}

AMERICAN PUBLIC HEALTH ASSOCIATION (US), AMERICAN WATER WORKS ASSOCIATION (US), WATER POLLUTION CONTROL FEDERATION (US). Physical and chemical examination of natural and treated waters in the absence of gross pollution / Nitrogen (Nitrate). Taras MJ, Greenberg AE, Hoak RD, Rand MC, editores. Standard Methods for the Examination of Water and Wastewater. 13th ed. Washington (DC): American Public Health Association US), American Water Works Association (US), Water Pollution Control Federation (US); 1971. p. 237-9.

ARRUDA, A.M.C.T. et al., Qualidade das Águas das Principais Fontes Naturais Urbanas em Garanhuns - PE., XVII Simpósio Brasileiro deRecursos Hídricos. São Paulo. SP. 2007.

ALABURDA, J. \& NISHIHARA L., Presença de composto de nitrogênio em águas de poços., Rev. Saúde Pública, São Paulo, v.32, n. 2, p. 531-7, Abril 1998.

FÁBIO ALEXANDRE COSTA MOTA, desenvolvimento de um fotômetro com fins didáticos, Universidade Federal do Amazonas, Instituto de Ciências Exatas, Programa de pós-graduação em química, dissertação, manaus, 2010.

LUCILIA MARIA PARRON; DAPHNE HELOISA DE FREITAS MUNIZ; CLAUDIA MARA PEREIRA. Manual de procedimentos de amostragem e análise físico-química de água. Primeira Edição, Embrapa Florestas, Colombo, PR, 2011, CDD 546.22 (21. Ed.).

MARCO A. M. SOUTO, MARINA M. OKADA, ISAURA A. OKADA, SÉRGIO, DOVIDAUSKAS. A determinação de nitrato em águas por espectrofotometria UV: usos e precauções. Rev Inst Adolfo Lutz, 65(1):66-70, 2006. 